\title{
Relación entre la responsabilidad social corporativa y las multinacionales
}

\author{
Partnership between corporate social responsibility and multinationals
}

Recibido: 12-05-2020 • Aprobado: 29-10-2020 • Página inicial: 47 - Página final: 68

\author{
Diana Marcela Restrepo Úsuga* \\ Jhon Edinson Mosquera Urrego**
}

\begin{abstract}
Resumen: la Responsabilidad Social Corporativa (RSC) consiste en el desarrollo por parte de la empresa, de acciones sociales que generen impacto a sus grupos de interés, las cuales se adaptan según el lugar donde se establezca la actividad económica. El presente artículo de reflexión, analiza la relación entre la responsabilidad social corporativa y las empresas multinacionales; se presenta el estado del arte donde se realiza una revisión bibliográfica analizando la evolución de la RSC y la forma como la vienen implementando las multinacionales en beneficio de los diferentes grupos de interés o stakeholders y de los lugares donde desarrollan sus actividades.
\end{abstract}

Palabras clave: Responsabilidad Social Corporativa (RSC); grupos de interés; stakeholders; multinacionales.

\begin{abstract}
Corporate Social Responsibility (CSR) consists of the development by the company of social actions that generate an impact on its stakeholders, which are adjusted according to where the economic activity is established. This reflection article analyzes the relationship between corporate social responsibility and multinational companies; the state of the art is presented where a bibliographic review is carried out analyzing the evolution of CSR and the way in which the multinationals are implemented to benefit the different interest groups or stakeholders and the places where they carry out their activities.
\end{abstract}

Keywords: Corporate Social Responsibility; interest groups; stakeholders; multinationals.

JEL: M14 - F23

\footnotetext{
* Profesional en Negocios Internacionales del Tecnológico de Antioquia, Especialista en Finanzas del Instituto Tecnológico Metropolitano de Medellín y Estudiante de MBA de la Universidad de Alicante, España. dianaru0710@gmail.com

ORCID: https://orcid.org/0000-0001-7918-7880

** Profesional en Administración Financiera del Tecnológico de Antioquia, Especialista en Finanzas y docente universitario del Tecnológico de Antioquia, Medellín - Colombia. admedinsonurrego@gmail.com ORCID: https://orcid.org/0000-0001-5007-4778
} 


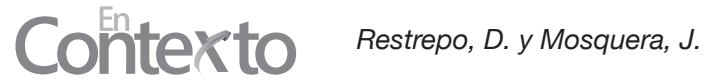

\section{Relação entre responsabilidade social corporativa e multinacional}

Resumo: Responsabilidade Social Corporativa (RSE) consiste no desenvolvimento pela empresa de ações sociais que geram impacto em seus stakeholders, as quais são ajustadas de acordo com o local onde a atividade econômica é estabelecida. Este artigo de reflexão analisa a relação entre responsabilidade social corporativa e empresas multinacionais; O estado da arte é apresentado onde é realizada uma revisão bibliográfica, analisando a evolução da RSE e a maneira como as multinacionais são implementadas para beneficiar os diferentes grupos de interesse ou partes interessadas e os locais onde realizam suas atividades.

Palavras-chave: responsabilidade social corporativa; grupos de interesse; partes interessadas; multinacionais. 


\section{Introducción}

La Responsabilidad Social Corporativa (RSC) es una forma de dirigir las empresas, está basada en la gestión de los impactos que su actividad genera sobre los clientes, empleados, accionistas, comunidades locales, medioambiente y sobre la sociedad en general (Observatorio de Responsabilidad Social Corporativa, 2019).

Desde el punto de vista académico, es de gran importancia analizar las medidas implementadas y el impacto que generan en cuanto a la relación empresasociedad en los mercados (países), en donde desarrollan su actividad económica las organizaciones; además de conocer los beneficios que ha traído en cuento al mejoramiento y posicionamiento de las empresas. Según los autores Knox \& Maklan (2004), se demuestra en la evidencia empírica que las acciones de la RSC tienen un impacto sobre la reputación de la empresa y sobre sus grupos de interés como lo son accionistas, directivos, empleados, clientes, proveedores, administraciones públicas, inversores y comunidad local.

La RSC funciona como un medio, en el cual se construyen actividades recíprocas entre empresas y la comunidad en general, es por ello que las empresas multinacionales se apoyan de dichas relaciones para promover su crecimiento y reconocimiento, en búsqueda de una sostenibilidad empresarial, generando valor a sus accionistas y crecimiento al mercado.

El escenario socioeconómico del mundo ha planteado un nuevo significado entre la relación que existe en la empresa y la sociedad, donde ahora se tiene como fin realizar un vínculo entre los aspectos sociales, ambientales y los objetivos económicos-financieros de las empresas, con el objetivo de generar prácticas socialmente responsables que generen impactos en el bienestar de todos aquellos individuos involucrados en los procesos que desarrolla la empresa.

Durante las últimas décadas, el concepto de responsabilidad social corporativa creció en importancia a nivel mundial (Bouslah et al., 2018), es por esto que el tema sobre RSC es uno de los que más crea debate, debido a la diversidad de enfoques con los que cuenta, enfatizando principalmente en la importancia de su aplicación y las consecuencias para la empresa y zona en donde esta se sitúa.

Según el Informe sobre las Inversiones en el Mundo (Naciones Unidas, 2019), aunque las multinacionales han tenido que lidiar con nuevos retos entre los que se encuentra la nueva revolución industrial, han sabido adaptarse, implementando 


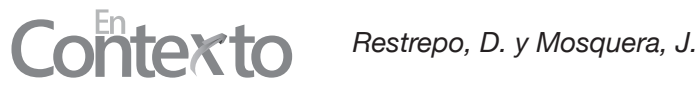

y desarrollando estrategias de responsabilidad social que contribuyen al desarrollo sostenible, en materia social, ambiental y de comunidad. Sin duda alguna, el sector empresarial posee un papel importante en el avance de la sociedad; tanto en términos económicos como sociales.

En relación con lo anterior, el presente artículo plantea analizar la relación entre la responsabilidad social corporativa y las empresas multinacionales; el texto partirá de una revisión de la literatura disponible, iniciando con la evolución que han tenido los términos tanto de RSC como de multinacionales; después se analizará los aspectos más importantes que las empresas multinacionales deben tener en cuenta a la hora de aplicar su RSC, información recopilada de escritos como fuente secundaria de información que permite incluir aspectos ya abordados en otros estudios sobre este tema.

\section{Las empresas multinacionales y la RSC}

\section{Empresas multinacionales}

Estas empresas aparecen en la segunda mitad del siglo XX y son consideradas como sociedades que operan en más de un país. Vale la pena resaltar en este apartado algunas de las definiciones que dan distintos autores al término de empresas multinacionales, las cuales se observarán en la Tabla 1.

Tabla 1

Definiciones de empresa multinacional

\begin{tabular}{cl}
\hline Autores & \multicolumn{1}{c}{ Concepto } \\
\hline Dunning (1981) & $\begin{array}{l}\text { Las empresas multinacionales son aquellas que tienen } \\
\text { una de sus plantas generadoras de ingresos localizadas } \\
\text { fuera del país de origen. }\end{array}$ \\
\hline Evans \& Newnham (1998) & $\begin{array}{l}\text { Es una organización creadora de ganancias que controla } \\
\text { bienes al menos en dos países. }\end{array}$ \\
\hline Willetts (2001) & $\begin{array}{l}\text { Es una compañía con base en un país, la cual tiene } \\
\text { filiales que mantienen tratos comerciales con una } \\
\text { sociedad o gobierno en otro país. }\end{array}$ \\
\hline Durán (2001) & $\begin{array}{l}\text { Es aquella que posee (coordina y controla) filiales en } \\
\text { uno o más países extranjeros. }\end{array}$ \\
\hline Pla Barber y León (2004) & $\begin{array}{l}\text { Es una empresa que tiene presencia significativa en todo } \\
\text { el mundo. }\end{array}$ \\
\hline
\end{tabular}




\begin{tabular}{cl}
\hline Autores & \multicolumn{1}{c}{ Concepto } \\
\hline \multirow{2}{*}{ Jones (2005) } & $\begin{array}{l}\text { Se entiende por empresas multinacionales las firmas que } \\
\text { controlan operaciones o activos que generan ingresos } \\
\text { en más de un país. }\end{array}$ \\
\hline & Es una organización económica que evoluciona desde \\
& su origen nacional hasta expandirse más allá de sus \\
& fronteras. La expansión se da a través de la inversión \\
extranjera directa, enfocada en explotar las capacidades & desarrolladas a nivel local. Siendo una de las ventajas \\
Quesada Chaves (2014) las empresas multinacionales, poseer un mayor & número de clientes potenciales, y una desventaja, que \\
& estas empresas se encuentran sujetas a más leyes y \\
& regulaciones que las empresas locales. \\
\hline
\end{tabular}

Elaboración propia.

Como se observa en la Tabla 1, en las definiciones anteriores existe un común denominador, que es ejercer una actividad empresarial en el exterior y tener presencia en al menos dos países, uno de origen, en donde pertenece la empresa, y un país de destino, que es aquel en donde tiene sus filiales.

\section{Evolución de la RSC}

El concepto de Responsabilidad Social Corporativa (RSC), se empezó a utilizar de manera generalizada a finales de la década de los cincuenta y principios de los años sesenta en los Estados Unidos de América, país que en aquella época comienza a reconocer la responsabilidad de las empresas privadas frente a aspectos derivados de su actividad empresarial como los riesgos laborales, la contaminación, entre otros (Belda-Calatayud, 2016).

Desde el siglo XIX y principios del XX, se encuentran algunos autores que ya hablaban de responsabilidad social. Una de las primeras definiciones la hace el autor Howard Bowen (1953), en su libro Social Responsibilities of the Businessman, el cual definía la RSC como las obligaciones que tenían los empresarios para seguir políticas, tomar decisiones o adoptar líneas de acción deseables en términos de los objetivos y valores de la sociedad. A continuación, en la Tabla 2, se muestra una línea del tiempo de la evolución de la RSC. 
Tabla 2

Evolución de la RSC

\begin{tabular}{|c|c|}
\hline Año & Acontecimiento \\
\hline 1919 & $\begin{array}{l}\text { Nace la Organización Mundial del Trabajo (OIT). En pro de intervenir } \\
\text { en los asuntos relativos al trabajo y las relaciones laborales, en más de } \\
190 \text { naciones, representó el puntapié de la responsabilidad corporativa } \\
\text { moderna a nivel global. }\end{array}$ \\
\hline 1945 & $\begin{array}{l}\text { Creación de la Organización de las Naciones Unidas (ONU). Es la } \\
\text { mayor agrupación internacional existente, interviene en el marco de la } \\
\text { Responsabilidad Social Empresarial (RSE), en el desarrollo económico, } \\
\text { social y los derechos humanos. }\end{array}$ \\
\hline 1953 & $\begin{array}{l}\text { H. Bowen Publica el primer libro en el que se desarrolla la idea de la } \\
\text { Responsabilidad Social Corporativa. }\end{array}$ \\
\hline 1971 & $\begin{array}{l}\text { Surge Green Peace. Esta organización es de las más reconocidas en todo } \\
\text { el planeta, trabaja para defender el medio ambiente y cuenta con oficinas } \\
\text { en } 43 \text { países de todos los continentes. }\end{array}$ \\
\hline 1976 & $\begin{array}{l}\text { Declaración sobre Inversión Internacional y Empresas Multinacional } \\
\text { (OCDE). }\end{array}$ \\
\hline 1982 & $\begin{array}{l}\text { Se delega en la segunda cumbre del planeta, la Comisión Mundial del } \\
\text { Medio Ambiente; se usa por primera vez el termino Desarrollo Sostenible. }\end{array}$ \\
\hline 1984 & E. Freeman propone la teoría de los Stakeholders o grupos de interés. \\
\hline 1987 & $\begin{array}{l}\text { Informe Brundtland y nacimiento del desarrollo sostenible. } \\
\text { Creación de la Comisión Mundial de Medio Ambiente y Desarrollo. }\end{array}$ \\
\hline 1990 & $\begin{array}{l}\text { Se crea la idea de Responsabilidad Social Empresarial y la valoración } \\
\text { del Talento Humano en las organizaciones, esto trae como consecuencia } \\
\text { la aparición de sindicatos y así mismo la evolución del área social, } \\
\text { económica, penal entre otras. La Unión Europea y Latinoamérica le dan } \\
\text { gran importancia y empiezan también a implementar la RSE. }\end{array}$ \\
\hline 1993 & Certificación Ambiental Norma ISO 14000. \\
\hline 1997 & $\begin{array}{l}\text { Publicación de la norma SA8000 de Responsabilidad Social. } \\
\text { Creación del GRI, Iniciativa de Reporte Global. }\end{array}$ \\
\hline 2000 & $\begin{array}{l}\text { La Unión Europea publica el Libro Blanco sobre Responsabilidad } \\
\text { Ambiental. } \\
\text { Firma de los Objetivos de Desarrollo del Milenio de la ONU. }\end{array}$ \\
\hline 2001 & La Comisión Europea publica el Libro Verde. \\
\hline 2003 & $\begin{array}{l}\text { Normas sobre la Responsabilidad de las Empresas Transnacionales y } \\
\text { otras Empresas Comerciales de Naciones Unidas. }\end{array}$ \\
\hline
\end{tabular}




\begin{tabular}{cl}
\hline Año & \multicolumn{1}{c}{ Acontecimiento } \\
\hline 2005 & $\begin{array}{l}\text { Implementación del protocolo de Kioto. Es un protocolo de la Convención } \\
\text { Marco de Naciones Unidas sobre el cambio climático que busca medidas } \\
\text { contra la contaminación. }\end{array}$ \\
\hline 2007 & $\begin{array}{l}\text { El parlamento europeo aprueba la resolución "RSC: Una nueva } \\
\text { asociación". }\end{array}$ \\
\hline 2019 & $\begin{array}{l}\text { Hoy en día la RSE, aunque no es obligatoria en la mayoría de las } \\
\text { organizaciones, la implementan y esta se encuentra en constante mejora } \\
\text { y desarrollo. }\end{array}$ \\
\hline
\end{tabular}

Aldeanueva, 2014.

Según los autores Knox \& Maklan (2004), se demuestra en la evidencia empírica, que las acciones de la RSC tienen un impacto sobre la reputación de la empresa, en cuanto a su know how, tanto positivos como negativos, como reconocimiento de la marca, buena o mala reputación de la empresa y sobre sus grupos de interés o stakeholders, estos grupos son de carácter interno y externo (Tabla 3), de acuerdo con sus necesidades de información y desarrollo por parte de la empresa multinacional. Esto indica que las empresas no solo deben velar por la maximización de sus beneficios, sino contribuir con el desarrollo de sus stakeholders.

\section{Tabla 3}

\section{Grupos de interés o stakeholders}

\begin{tabular}{|c|c|}
\hline Tipos de grupos de interés & Denominación \\
\hline \multirow{2}{*}{ Internos } & Accionistas \\
\hline & Empleados \\
\hline \multirow{8}{*}{ Externos } & Clientes \\
\hline & Proveedores \\
\hline & Competidores \\
\hline & Agentes sociales \\
\hline & Administraciones públicas \\
\hline & Comunidad local \\
\hline & Sociedad y público en general \\
\hline & Medio ambiente y generaciones futuras \\
\hline
\end{tabular}

Lizcano y Moneva, 2004. 


\section{Contex to Pestrepo, D. . y yossuere,, .}

Otra de las definiciones en la cual se puede apoyar este trabajo, es la planteada por los autores Bondy et al. (2008), quienes explican que la RSC engloba un conjunto heterogéneo de políticas y acciones que promueven las empresas de forma voluntaria con el objetivo de satisfacer las expectativas de los grupos de interés. Desde otra perspectiva, la RSC se entiende como el deber que asumen las empresas de compensar el efecto negativo que generan sus actividades en el lugar donde se sitúan, lo anterior se soporta con la definición que hace el autor Sethi (2009), el cual expone que la RSC refleja la percepción que tienen los directivos de los efectos sociales y medioambientales de las actividades que realizan sus empresas y las restricciones que se imponen voluntariamente para amortiguarlas.

Siguiendo con más definiciones, se encuentra que la RSC pide a las empresas una actuación de "buen gobierno corporativo" tanto al interior como al exterior de la misma. Un "buen gobierno corporativo" significa mantener buenas relaciones con su personal, tener transparencia en sus operaciones, respeto por los consumidores y preocupación por el medio ambiente (Kliksberg, 2006).

Así mismo, se habla que la RSC se entiende como el conjunto de medidas y acciones económicas, sociales y ambientales que llevan a cabo las empresas $\mathrm{u}$ organizaciones hacia sus stakeholders internos (directivos, ejecutivos, trabajadores) y externos (proveedores, acreedores, comunidad, gobierno, medios, sociedad civil), de manera proactiva y en orden no solo de cumplir con sus obligaciones sociales, sino de ir más allá de estas, contribuyendo a través del beneficio general y promoviendo el desarrollo económico incluyente (Jiménez-Peña, 2006).

En definitiva, se evidencia que el factor común entre las definiciones antes expuestas es que la RSC está siendo parte de un proceso de evolución que las empresas han desarrollado a lo largo del tiempo y que es promovida por la sociedad, que cada vez exige más claridad en todos los aspectos relacionados con el desarrollo de su entorno global. Es de anotar que las empresas multinacionales también tienen la oportunidad de ser reconocidas por ser un eslabón esencial en el crecimiento de la economía y por promover actividades que involucren de manera positiva a sus grupos de interés, obteniendo así beneficios para las partes.

\section{Relación entre empresas multinacionales, RSC y Gobierno}

Las empresas multinacionales están constituidas por una gran red de unidades empresariales que se extienden por varios países, las cuales son conocidas como sucursales o subsidiarias. A su vez, estas son coordinadas por un equipo 
localizado en el país de origen u oficina principal, lo que los obliga a tener varios grupos de interés alrededor de sus empresas, tanto en el país de origen como en el lugar de destino de sus sucursales, considerando además todos los órganos de gobierno que operan en estos lugares, como pueden ser los gobiernos locales, administradoras de entidades públicas, las ONG dedicadas al cuidado del medio ambiente o de las comunidades locales en estos países. Por lo anterior, las multinacionales no solo deben atender un número mayor de grupos de interés, sino que, además, deben cumplir con las expectativas de cada uno de ellos, las cuales al igual que los valores y las normas sociales son diferentes en cada país y van cambiando con el tiempo.

Por su parte, la globalización ha generado en las multinacionales la necesidad constante de cambio y adecuación de su filosofía; es por esto que los Estados han estipulado normas de conducta empresarial que minimicen los impactos sociales y medioambientales que la actividad económica de estas organizaciones genera a corto y largo plazo sobre su espacio geográfico, lo que contribuye a cambiar el modelo de dirección empresarial, el cual solo se enfocaba en la maximización del beneficio económico para los accionistas de la empresa.

El autor Sala i Martín (2006) expone que un flujo entrante de inversión proveniente de estas empresas puede estimular el desarrollo local gracias al aumento y mejora de recursos y capacidades (stock de capital, tecnología, capacidad empresarial y acceso a mercados), aumento de competencia, mejor asignación de recursos, desarrollo de recursos humanos, generación de empleo, etc.

En este sentido, organismos gubernamentales a nivel mundial han creado normativas que las empresas multinacionales deben cumplir para contrarrestar en gran medida los efectos que puedan causar por su actividad, una de ellas creada en el año 1996 fue la norma ISO 14001, la cual habla sobre la implantación de un Sistema de Gestión Medioambiental (SGMA); esta norma se estableció con el fin de concientizar a las empresas en el cuidado del medio ambiente, además de que su cumplimiento es certificable y otorgaba un reconocimiento a nivel internacional.

Adicional a esto, en el año 1997 se publica la primera norma sobre Responsabilidad Social denominada como SA 8000 (Social Accountability International [SAI]), se funda el Global Reporting Initiative (GRI) y el World Business Council for Sustainable Development (WBCSD) crea un grupo de trabajo sobre Responsabilidad Social Corporativa (RSC) y en 1999 se crea el Dow Jones Sustainability Index (DJSI), primer índice bursátil que registra el rendimiento de las empresas líderes en sostenibilidad y además se crea el Pacto 


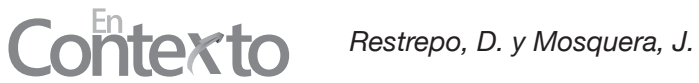

Mundial de las Naciones Unidas (Global Compact), adoptando unos principios básicos para la construcción de los pilares de una sociedad más responsable, necesarios para mantener la nueva economía global (Sánchez et al., 2010).

Algunos expertos (Villareal, 2004; Sala i Martín, 2006) consideran la inversión extranjera directa (IED) como el motor del crecimiento económico de un país, dentro de la cual sus principales beneficios son: la transferencia de tecnología, la inversión financiera y la generación de empleo. También exponen que puede generar competencia en el mercado y fomentar la creación de un ambiente empresarial competitivo al incrementar el progreso de los negocios y el crecimiento de los lugares donde se establecen las organizaciones, lo que es visto como una contribución al desarrollo del capital humano y de la comunidad. Es de gran importancia la buena relación entre las empresas y los gobiernos del país donde se sitúan las sucursales, teniendo en cuenta que ambos buscan beneficiarse en términos monetarios, las empresas multinacionales favorecen a los países que ingresan, respecto a generación de empleo, ingresos directos por pago de impuestos, entre otros. Por su parte, estas empresas aumentan su cobertura de mercado y acceden a mano de obra calificada a costos que les permita ser competitivos y excedentes favorables para su operación.

Tal como se mencionó anteriormente, las expectativas de los grupos de interés pueden diferenciarse tanto en el tiempo como en el espacio, lo que obliga a las empresas a desarrollar iniciativas de RSC que se adapten a las condiciones de cada país. Además de esto, tienen la obligación de proyectar en su informe de gestión anual las memorias de RSC con toda la información completa de los lugares donde se ejerce su actividad.

En este sentido, la IED que realizan las multinacionales, ayuda al mejoramiento continuo de los recursos con los que cuentan los países donde se desarrolla la actividad económica, además de que potencia la generación de empleo beneficiando a la comunidad local, que, al contratar personal de esta, dará capacitación en el uso de los métodos de producción y en el uso de tecnologías que sean empleadas para su desarrollo.

Por todo lo anterior, se puede inferir que las empresas multinacionales han tenido efectos positivos en los países de destino, y se han caracterizado por generar empleo y contribuir con el desarrollo económico de los Estados donde se establecen para el desarrollo de sus actividades. 


\section{Responsabilidad Social Corporativa (RSC) en el éxito competitivo de las multinacionales}

Son numerosos los beneficios derivados de la adopción de acciones socialmente responsables en todos los ámbitos empresariales, tales como la satisfacción de los recursos humanos, aspectos financieros o ventajas comerciales, por ejemplo. Pero en la base de cualquiera de estos beneficios está la obtención de ventajas competitivas. Es decir, basados en la teoría, se puede decir que las empresas que aplican acciones socialmente responsables logran competir en mejores circunstancias en el mercado y los lleva a tener éxito en el cumplimiento de sus objetivos empresariales.

Cuando una empresa asume voluntariamente el compromiso de la RSC, va más allá de obligaciones reglamentarias, está adoptando un modelo de negocio abierto, uniendo intereses de diversos agentes y consiguiendo calidad y viabilidad, lo que supone que la reputación de las empresas se empiece a configurar como un valioso intangible, capaz de generar ventajas competitivas sólidas y sostenibles en el tiempo (Lizcano y Moneva, 2004).

Autores como Nieto Antolín y Fernández Gago (2004) exponen que muchas empresas han decido aceptar la nueva concepción de la empresa y las empresas más representativas han formalizado sus prácticas en materia de RSC y las difunden habitualmente. Diversas investigaciones han expuesto las causas de esta generalización, entre los cuales podemos destacar: la presión reguladora e iniciativas promovidas por gobiernos, organizaciones de empresas e instituciones y organismos internacionales (OCDE, UE, ONU).

La relación entre la RSC y el valor empresarial ha sido objeto de estudio en varias investigaciones, según lo observado en fuentes bibliográficas, donde existen áreas en las que las operaciones de RSC son reconocidas como apoyo en la mejora del rendimiento organizacional de las empresas; estas mejoras se reflejan en cuanto a la relación empresa-consumidor, mayor facilidad para conseguir financiación y atracción de talento competitivo. Más concretamente, el éxito competitivo ha sido señalado como un logro clave tras la realización de acciones de RSC, entendiendo por éxito la obtención de unos resultados positivos para la empresa en términos de posicionamiento en el mercado y que van más allá del ámbito financiero (Fernández-Kranz \& Santaló, 2010).

La aplicación de la RSC en las multinacionales también hace parte del proceso de generación de reputación y valor que desarrollan las empresas, debido a que 


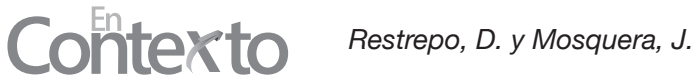

estas invierten en su RSC con un doble propósito, por una parte, contribuyen con el desarrollo y progreso del entorno y, por otra parte, ayudan en la proyección de imagen que se refleja a sus stakeholders.

Según lo anterior, la RSC tiene incidencia sobreelnombre de la empresa, sureputación y los efectos financieros al realizar inversiones socialmente responsables, además de que la aplicación de la RSC en las multinacionales, ya sea por presión debido al aumento de la regulación (por parte de gobiernos, organizaciones de empresas e instituciones y organismos internacionales), o por la presión del mercado (con unos consumidores más exigentes) o la presión del mercado financiero (generación de mayores benéficos por parte de accionistas), hace que estas empresas obtengan un valor agregado y se diferencien de su competencia.

Para hablar de los beneficios de la RSC y la reputación de la empresa, los autores Knox \& Maklan (2004) exponen que los efectos finales de la RSC sobre la reputación corporativa se pueden dividir en cuatro categorías: ganancias, riesgo asociado a la pérdida de las ganancias, riesgo de costo y reducción de costos (Figura 1).

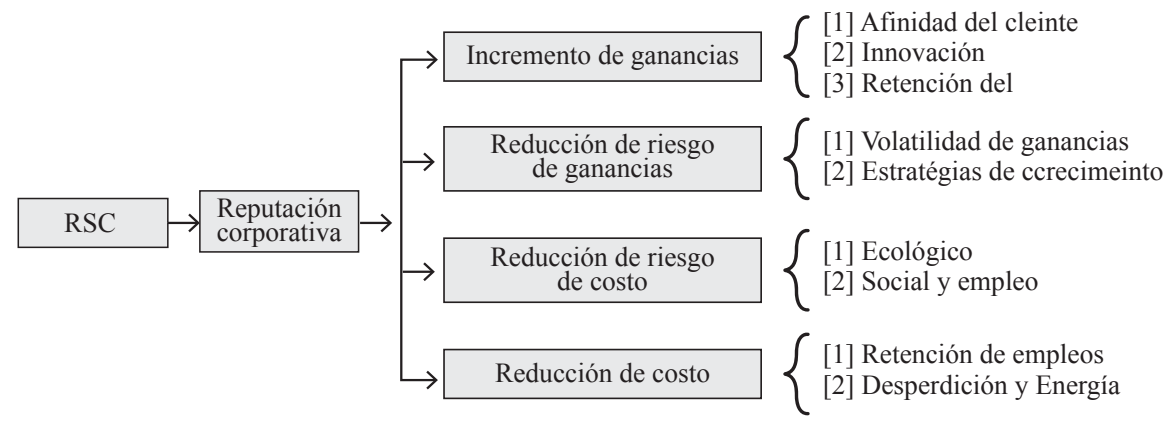

Figura 1. Efectos de la relación entre la RSC y la reputación corporativa.

Knox \& Maklan (2004).

Según los autores García y Llorente (2009), en el concepto de reputación corporativa que ha ido desarrollando los diferentes expertos en el tema, tanto procedentes del mundo académico como procedentes del mundo de la consultoría, se han repetido dos ideas constantes:

- Que la reputación corporativa de una empresa es consecuencia de una serie de relaciones eficaces y comprometidas con los diferentes stakeholders de esa empresa. 
- Que la reputación corporativa se identifica con un estudio de consolidación definitiva de la imagen corporativa positiva de esa empresa.

Es de aportar que el incremento de la iniciativa de las empresas en los aspectos relacionados con la RSC viene evolucionando constantemente; es por esto que las empresas multinacionales comenzaron a formalizar dentro de sus procesos las prácticas en materia de RSC y su difusión mediante los medios de comunicación.

En la actualidad, la mayor parte de las empresas listadas en Fortune 500, la cual exhibe de forma anual las 500 mayores empresas estadounidenses de capital abierto a cualquier inversor, según su volumen de ventas, describen en su página web sus logros en materia de RSC y casi la mitad de las incluidas en el Fortune 250 elaboran informes específicos sobre aspectos sociales y medioambientales de sus actividades (Fortune, 2019). También se ha comprobado que las diez empresas más admiradas del mundo, según el ranking de Merco (2019), desarrollan prácticas de RSC y las difunden para aprovechar los efectos positivos que genera la transparencia informativa.

Además de esto, según el artículo publicado en la Revista AECA, titulado Marco Conceptual de la Responsabilidad Social Corporativa (Lizcano y Moneva, 2004), la dirección estratégica de la empresa debe ir orientada a satisfacer necesidades diversas, y a veces contrapuestas, de los distintos grupos de interés, introduciendo factores diferenciales de innovación que, desarrollados adecuadamente con los aspectos de dirección y gestión, otorgan a la empresa ventajas competitivas duraderas.

Se puede afirmar entonces que la RSC es una nueva forma de hacer negocios, donde se trata de hacer una integración de los aspectos sociales, económicos y medioambientales con la actividad propia que desarrolla la empresa y que sean de gran aporte al éxito de la misma.

Por su parte, el desempeño con clientes, con proveedores y con los empleados en la gestión de la cadena de valor son importantes para la obtención de ventajas competitivas (Kusyk \& Lozano, 2007). Esto quiere decir que la RSC tiene una influencia positiva sobre la apreciación que hace el cliente al realizar una compra y sobre la competitividad en la calidad de los productos que ofrece las empresas, las cuales apuestan por productos que satisfagan a los clientes y que contribuyan con el desarrollo sostenible; además de esto, la RSC aporta en la gestión que se realiza con los empleados, lo que lleva a mejorar su motivación $\mathrm{y}$ rendimiento laboral. 


\section{Contex to Pestropo, D. .y Mossueres,.}

Es así como Nieto Antolín (2008) expone que las prácticas de RSC también pueden ayudar a reforzar los recursos internos de las empresas y mejorar la calidad del contexto competitivo en el que operan. Se ha reconocido que las empresas que orientan las prácticas de RSC hacia sus empleados mejoran el clima laboral, reducen el absentismo y los costes derivados de los conflictos laborales. Estas empresas resultan más atractivas ante sus empleados y, por ello, tienen una capacidad superior para atraer y conservar los recursos humanos más competentes.

Adicionalmente, las prácticas de responsabilidad con proveedores, clientes y la comunidad local inciden directamente sobre aspectos clave del contexto competitivo de las empresas, ya que mejoran la oferta de "inputs" o recursos especializados de alta calidad, potencian una demanda sofisticada y exigente, crean un entorno más productivo y transparente y mejoran los sectores relacionados y complementarios (Porter \& Kramer, 2002).

Finalmente y según los autores Prahalad \& Hammond (2002) que consideran que se puede obtener beneficios de las actuaciones sociales; las empresas pueden obtener ventajas competitivas si se dedican a atender a sectores de menos recursos económicos, y así obtener una nueva fuente de crecimiento de ingresos, una mayor eficiencia y el acceso a la innovación para ayudar a dichos sectores. Por consiguiente, estos factores pueden considerarse como una diferenciación, una ventaja competitiva y un liderazgo en el sector donde desarrollen su actividad estas empresas.

¿Qué pueden hacer los gobiernos para promover la responsabilidad social de las empresas en sus territorios?

La RSC se fundamenta en una visión global de la empresa, y se tiene claro que esta no solo se aplica por ser social o amable con los grupos de interés. La RCS debe estar integrada en la visión y la estrategia global de la empresa y sus stakeholders.

Según el autor Vilanova y Dinarés (2009), la matriz conceptual de la RSC que deben ejercer todas las empresas es la que se muestra en la Figura 2. 


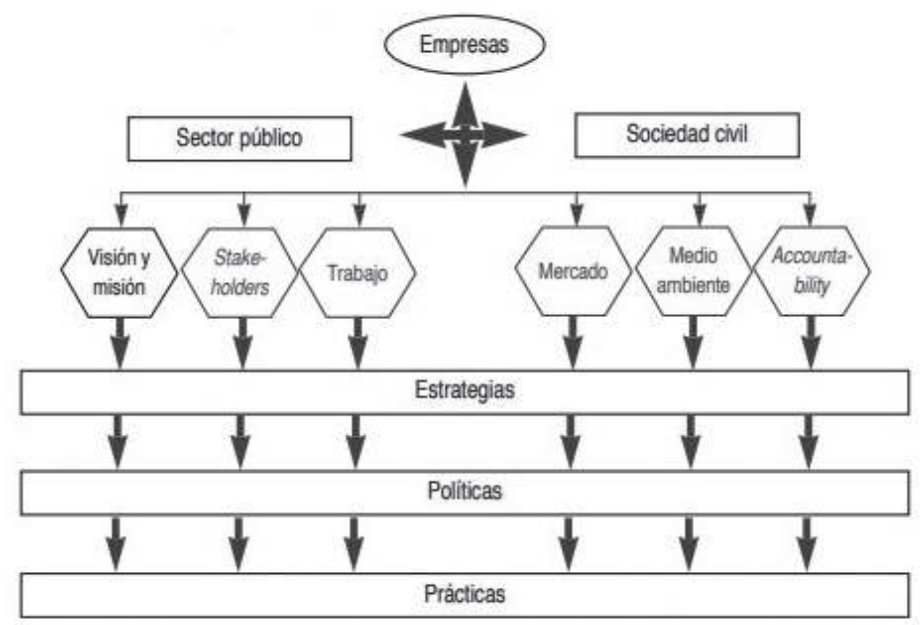

Figura 2. Matriz conceptual de la RSC.

Vilanova y Dinarés, 2009.

Desde esta perspectiva, cabe entender que actualmente la RSC esté presente en los planes de gobierno de los países, es por esto por lo que sus gobernantes se han tenido que enfrentar a un desafío social importante, puesto que se refleja el papel de la empresa en el proceso de desarrollo económico y su contribución en la consecución de los retos sociales y medioambientales que se plantean.

En el contexto europeo, el Libro Verde, Fomentar un marco europeo para la responsabilidad social de las empresas, y la comunicación La responsabilidad social de las empresas: una contribución empresarial al desarrollo sostenible, publicados por la Comisión Europea, se han convertido en la referencia cuando se trata de enfocar esta cuestión (Comisión Europea, 2001).

En la aproximación a la RSC que se hace en cada país se condensan varios elementos: la estructura política e institucional, el estilo y la organización de los procesos políticos, la estructura social, la intensidad con que se asume la actitud de voluntariedad, la manera como se describe el papel de las empresas en el ámbito local y nacional, el papel que juegan las ONG y las sociedades civiles, las características del sistema educativo y los valores que se transmiten en él, lo que se espera de los líderes y las tradiciones históricas (Lozano et al., 2005). Lo anterior, hace que la RSC ya no se refiera simplemente a la relación empresa-sociedad, sino que se vea como una manera actual de ver el papel de la empresa en la sociedad, donde se incorporan los elementos desde la perspectiva 


\section{Contex to Pestrepo, D. . y wossuera,,.}

de gobierno y sostenibilidad y hace que cada vez sea más necesario que las empresas y los países tengan una clara conciencia de la necesidad de elaborar políticas de RSC que aporten al desarrollo sostenibles y apoyen los Objetivos de Desarrollo Sostenible (ODS), los cuales quieren erradicar la pobreza, proteger el planeta y asegurar la prosperidad a nivel global.

Los ODS agregan elementos que van muy de la mano con la RSC, es decir, la RSC que realizan las empresas, en este caso las multinacionales, aportan al cumplimiento de los objetivos mundiales, ya que en su diseño integran tres dimensiones importantes como son: lo económico, lo social y lo ambiental, dando prioridad a la reducción de la pobreza y el hambre, a la defensa de los derechos humanos, al empoderamiento de la mujer como igualdad de género; además de contribuir con la reducción de las desigualdades dentro de cada país y apoyando el consumo sostenible por parte de la población en general.

Los ODS al igual que la RSC tienen como función aportar al crecimiento económico y sostenible de todos los países, que a su vez sea respetuoso con el medio ambiente y la población. Según Gómez Gil (2017/18), un elemento innovador de los ODS es su carácter universal de aplicación en todo el mundo y para todos los países, con una visión holística e interrelacionada en la que cada objetivo se superpone y refuerza mutuamente, con una actuación multinivel simultánea en los espacios locales, regionales, nacionales y globales, apostando por construir una solidaridad global.

En el desarrollo de las políticas públicas sobre RSC es muy importante el contexto social, cultural y político de cada país, según los autores Lozano et al. (2005), se puede destacar que mientras en unos países las políticas de RSC se han definido partiendo de los temas sociales y generando una política pública independiente, en otros, la acción gubernamental para la RSC se ha incorporado a la política nacional de sostenibilidad. En este sentido, se centra más en el concepto de desarrollo sostenible.

En algunos países, la RSC no es solo una cuestión conceptual, sino una opción política donde se vincula directamente empresa y sociedad, y la relación entre estos actores sociales y sus políticas de negociación colectiva. La RSC no debe estar desvinculada de los retos políticos y económicos actuales, sino al contrario, debe estar inmersa en los nuevos cambios que ha traído la globalización. De esta forma, se presenta a la RSC como un nuevo modelo de gobernabilidad vinculado a la competitividad nacional y al cumplimiento de los objetivos mundiales. 
En última instancia, la acción gubernamental en el campo de la RSC no debe plantearse solo como un debate sobre políticas públicas, sino que los gobiernos deben incluirlo en un contexto mucho más amplio, como un debate sobre modelo de gobernanza, en el marco de la crisis del Estado de bienestar y los nuevos retos generados por la economía globalizada (Lozano et al., 2005).

Finalmente, se evidenció a través del proceso de investigación y consulta que la RSC hace parte de un proceso de evolución que las empresas han desarrollado a lo largo del tiempo y que cada vez es más promovida por la sociedad, la cual exige constantemente más claridad en todos los aspectos relacionados con el desarrollo de su entorno global. Así mismo, se refleja que el papel de las empresas multinacionales también debe ser reconocido, pues son un eslabón esencial en el crecimiento de la economía, al promover actividades que involucran de manera positiva a sus grupos de interés obteniendo así beneficios para las partes.

Igualmente, se pudo constatar que la RSC no es solo una cuestión conceptual, sino una opción política, donde se vincula directamente la empresa y la sociedad y la relación entre todos sus actores sociales; es allí donde se evidencia que las políticas de RSC no deben estar desvinculadas de los retos políticos y económicos actuales de las empresas y los países donde estas se sitúan, sino que deben estar inmersas en los nuevos cambios que ha traído la globalización, donde se presenta la RSC como un nuevo modelo de gobernabilidad vinculado a la competitividad nacional y al cumplimiento de los objetivos de desarrollo mundial.

También se evidencia que cuando una organización asume facultativamente el compromiso de la RSC, se acoge a un modelo de negocio abierto, donde en su estrategia siempre están presente sus stakeholders, y donde se le da relevancia a la visión que tienen estos de la empresa, pues son ellos quienes están en constante contacto con los productos o servicios que las empresas desarrollan y son los encargados de proyectar el valor de la marca en la sociedad, lo que puede beneficiar o no a la empresa según la reputación que esta tenga frente a sus grupos de interés.

Otro de los puntos importante en el desarrollo del trabajo, es que se demuestra que la presión que han ejercido los nuevos consumidores también ha llevado a que las multinacionales realicen un mayor esfuerzo en mantener unas políticas de RSC coherentes, prácticas y que de verdad aporten al crecimiento y desarrollo de sus comunidades tanto a nivel social, laboral y medioambiental. 


\section{Contex to Pestrepo, D. . y yossuere,, .}

Así pues, gracias a que los consumidores somos más conscientes de los productos que adquirimos y de cómo se desarrollan, es lo que ha llevado a que las empresas se preocupen por mostrar prácticas de buen gobierno, donde se relacionen todos los aspectos que están dentro de la RSC, además de que estas le den un valor significativo a la elaboración de sus políticas y la toma de decisiones a la hora de invertir de una manera sostenible.

Las empresas multinacionales son conscientes de que su contribución al desarrollo sostenible depende en gran medida de cómo desarrollen sus operaciones dentro y fuera de la organización, además reconocen que son ellas quienes aportan al crecimiento económico y social de los lugares donde desarrollan su actividad, en este sentido, la importancia de la responsabilidad social para las organizaciones radica principalmente en el desarrollo de un sistema de gestión que beneficie tanto a la empresas como a sus empleados, clientes, proveedores accionistas, el medioambiente y la comunidad en general. Por otra parte, creo firmemente que a las empresas se les debe dar una oportunidad y no estar siempre pendiente de lo negativo, se debe ahondar más en las actividades que desarrollan para contrarrestar muchos de los daños ocasionados que realizan a las comunidades y al medioambiente, y creer en que se puede lograr un cambio en cuanto a la manera de hacer negocio de una forma sostenible y amigable con el medioambiente. Además, la RSC es una forma de crear valor a largo plazo que las empresas deben seguir desarrollando aún más para que esto se les convierta en el mejor intangible que poseen y puedan competir con base en criterios de calidad y buenas prácticas.

\section{Conclusiones}

Se evidenció a través del proceso de investigación que, la RSC hace parte de un proceso de evolución que las empresas han desarrollado a lo largo del tiempo que cada vez es más promovida por la sociedad, la cual exige constantemente más claridad en todos los aspectos relacionados con el desarrollo de su entorno global. Así mismo, se refleja que el papel de las empresas multinacionales también debe ser reconocido, pues son un eslabón esencial en el crecimiento de la economía, al promover actividades que involucran de manera positiva a sus grupos de interés obteniendo así beneficios para las partes.

Dada la investigación realiza, se puede concluir que las empresas multinacionales en un contexto global se constituyen como un factor importante para el desarrollo de las economías, dado que estas generan flujos de inversión de capital, en pro de apalancar un mercado específico y obtener beneficios de 
este, son potenciadoras de la unidad productiva y por lo general, crean grandes cantidades de empleos directos en el lugar al que acceden.

La RSC puede ser un tema controvertido, dado que de las multinacionales se espera que generen acciones en beneficio de los territorios donde se ubican, especialmente cuando su actividad económica se relaciona con la explotación de materias primas, según el informe de Corporate Excellence sobre gestión de intangibles (2019), la inversión sostenible ya es considerada una opción rentable. Actualmente existen carteras con paquetes ESG (por sus siglas en inglés Enviromental, Social, Governnence) que permiten a los inversionistas conocer y medir el impacto medioambiental y social de sus inversiones. Es por esto por lo que a los inversionistas les llama la atención las empresas que desarrollen buenas prácticas de gobierno, puesto que quiere decir que sus cuentas y estrategias de mercado pueden generar beneficios a largo plazo y satisfacer sus necesidades en cuanto a inversión; sin desconocer que también puede haber inversionistas que se interesen por los rendimientos y no en las buenas prácticas.

Por otra parte, las empresas multinacionales son conscientes de que su contribución al desarrollo sostenible depende en gran medida de como desarrollen sus operaciones dentro y fuera de la organización, además conocen que son ellas quienes aportan al crecimiento económico y social de los lugares donde desarrollan su actividad, en este sentido, la importancia de la responsabilidad social para las organizaciones radica principalmente en el desarrollo de un sistema de gestión que beneficie tanto a la empresas como a sus empleados, clientes, proveedores accionistas, el medioambiente y la comunidad en general. 


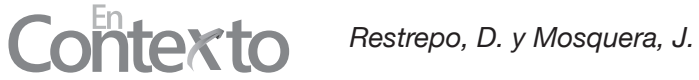

\section{Referencias}

Aldeanueva, I. (2014). Antecedentes y evolución histórica de la responsabilidad social corporativa. Strategos, 6(12), 75-82.

Belda-Calatayud, E. (2016). La responsabilidad social corporativa en el sector turístico español [Tesis de pregrado, no publicada]. Universidad Politécnica Valenciana.

Bondy, K., Matten, D., \& Moon, J. (2008). Multinational corporation codes of conduct: governance tools for corporate social responsibility?. Corporate Governance: An International Review, 16(4), 294-311.

Bouslah, K., Kryzanowski, L., \& M'Zal, B. (2018). Social performance and firm risk: Impact of the financial crisis. Journal of Business Ethics, 149, 643-669.

Bowen, H. (1953). Social responsibilities of the businessman. University of Iowa Press.

Comisión Europea. (2001). Libro verde. Fomentar un marco europeo para la responsabilidad social de las empresas. https://observatoriorsc.org/ libro-verde-fomentar-un-marco-europeo-para-la-responsabilidad-socialde-las-empresas/

Corporate Excellence. (2019). Somos un celerador de innovación, formación y conocimiento en reputación, marca, comunicación, sostenibilidad e intangibles. Corporate Excellence.

Durán, J. (2001). Estrategia y economía de las empresas multinacionales. Pirámide.

Evans, G., \& Newnham, J. (1998). The penguin dictionary of international relations. Penguin Reference.

Fernández-Kranz, D., \& Santaló, J. (2010). When necessity becomes a virtue: the effect of product market competition on corporate social responsibility. Journal of Economics \& Management Strategy, 19(2), 453-487.

Fortune. (2019). World's most admired companies. https://fortune.com/worldsmost-admired-companies/2020/search/?ordering=asc

García, M., y Llorente, C. (2009). La responsabilidad social corporativa: una estrategia para conseguir imagen y reputación. Icono 14, 7(2), 95-124.

Gómez-Gil, C. (2017/18). Objetivos de Desarrollo Sostenible(ODS): una revisión crítica. Papeles de relaciones ecosociales y cambio global, 140, 107-118. 
Jiménez-Peña, G. (2006). Más allá de la sesponsabilidad social empresarial: hacia un punto de vista de la ciudadanía corporativa deliberativa. Vox Populi, 5, 25-30.

Jones, G. (2005). Multinationals and global capitalism. From the nineteenth to the twenty-first century. Oxford University Press.

Kliksberg, B. (2006). Ética empresarial: ¿moda o demanda imparable?. http:// cyta.com.ar/biblioteca/bddoc/bdlibros/rse/334_as_etica_moda.pdf

Knox, S., \& Maklan, S. (2004). Corporate social responsibility: Moving beyond investment towards measuring outcomes. European Management Journal, 22(5), 508-516.

Kusyk, S., \& Lozano, J. (2007). SME social performance: A four-cell typology of key social issue drivers and barriers on social issues and their implitacions for stakeholder theory. Corporate Governance, 7(2), 229-239.

Lizcano, J., y Moneva, M. (2004). Marco conceptual de la responsabilidad social corporativa. Revista AECA, 68, 31-36.

Lozano, J., Albareda, L., y Tamyko, Y. (2005). ¿Qué pueden hacer los gobiernos para promover la Responsabilidad Social de la Empresa (RSE)?. CIRIEC, 53, 53-64.

Merco. (2019). Responsabilidad y gobierno corporativo. ttp://www.merco. info/es/ranking-merco-responsabilidad-gobierno-corporativo

Naciones Unidas. (2019). Informe sobre las inversiones en el mundo 2019. UNCTAD.

Nieto-Antolín, M. (2008). La difusión de las prácticas de responsabilidad social en las empresas multinacionales. Pecvnia, Monográfico, 33-64.

Nieto-Antolín, M., y Fernández Gago, R. (2004). Responsabilidad social corporativa, la ultima innovación en management. Universia Business Review, 5(1), 28-39.

Observatorio de Responsabilidad Social Corporativa. (2019). Observatorio de Responsabilidad Social Corporativa. https://observatoriorsc.org/larsc-que-es/

Pla-Barber, J., y León, F. (2004). Dirección de Empresas Internacionales. Pearson Educación. 


\section{Contex to Restrepo, D. y Mossuera, J.}

Porter, M., \& Kramer, M. (2002). The competitive advantage of corporate philanthropy. Harvad Business Review, 80(12), 56-68.

Prahalad, C., \& Hammond, A. (2002). Serving the world's poor, profitably. Harvard Business Review, 80(9), 48-57.

Quesada-Chaves, F. (2014). La red trampolín: multinacionales en Latinoamérica [Tesis de doctorado]. Universidad de Valencia. http://mobiroderic. uv.es/bitstream/handle/10550/35174/TESIS\%20DO\%20CTORAL\%20 (Federico\%20Quesada\%20Chaves).pdf?sequence=1\&is\%20Allowed=y

Sala i Martín, X. (2006). Globalización y reducción de la pobreza. FAES.

Sánchez, M., García, E., y Pardo, R. (2010). Análisis de herramientas de sostenibilidad y RSC para su aplicación a la indrustria de procesos. Estudios Empresariales, 2, 81-98.

Sethi, P. (2009). A conceptual framework for environmental analysis of social issues and evaluation of business response patterns. Academy of Management Review, 4(1), 29-56.

Vilanova, M., y Dinarés, M. (2009). Modelo de indicadores de RSE para pymes. Manual de uso. ESADE.

Villareal, C. (2004). Las teorías de la localización de la inversión extranjera directa: una aproximación. Innovaciones de Negocios, 1(2), 245-263.

Willetts, P. (2001). Transnational actors and international organizations in global politics, en John Baylis y Steve Smith, The globalization of world politics, an introduction to international relations. Oxford University Press.

\section{Para citar este artículo:}

Restrepo, D. y Mosquera, J. (2020). Relación entre la responsabilidad social corporativa y las multinacionales. En-Contexto, 8(13), 47-68. 